\title{
Erratum: Spins of primordial black holes formed in the matter-dominated phase of the Universe [Phys. Rev. D 96, 083517 (2017)]
}

Tomohiro Harada, Chul-Moon Yoo, Kazunori Kohri, and Ken-Ichi Nakao

(Q) (Received 11 March 2019; published 28 March 2019)

DOI: $10.1103 /$ PhysRevD.99.069904

In the 12th line of the Abstract, the prefactor $1.9 \times 10^{-6}$ in the estimate of $\beta_{0}$ should be replaced with $1.9 \times 10^{-7}$. The prefactor $1.894 \times 10^{-6}$ in both Eq. (5.8) and the upper line of Eq. (5.10) should be replaced with $1.921 \times 10^{-7}$. No result is affected except the above correction.

The authors thank Tommi Tenkanen and Takahiro Terada for bringing this point to the their attention. 